\title{
Electroacupuncture alleviates neuromuscular dysfunction in an experimental rat model of immobilization
}

\author{
Jun Yang ${ }^{1}$, Su Min ${ }^{1}$, Fei Xie ${ }^{1}$, Jingyuan Chen ${ }^{1}$, Xuechao Hao ${ }^{1}$ and Li Ren ${ }^{1}$ \\ ${ }^{1}$ Department of Anesthesiology, The First Affiliated Hospital of Chongqing Medical University, Chongqing 400016, China \\ Correspondence to: Su Min, email: sumin89011061@Sina.com \\ Keywords: electroacupuncture, nicotinic acetylcholine receptors, immobilization, skeletal muscle atrophy, neuromuscular function \\ Received: January 20, $2017 \quad$ Accepted: July 25, $2017 \quad$ Published: August 14, 2017 \\ Copyright: Yang et al. This is an open-access article distributed under the terms of the Creative Commons Attribution License 3.0 \\ (CC BY 3.0), which permits unrestricted use, distribution, and reproduction in any medium, provided the original author and source \\ are credited.
}

\section{ABSTRACT}

Immobilization-related skeletal muscle atrophy is a major concern to patients in Intensive Care Units and it has a profound effect on the quality of life. However, the underlying molecular events for the therapeutic effect of electroacupuncture to treat muscle atrophy have not been fully elucidated. Here we developed an immobilization mouse model and tested the hypothesis that skeletal muscle weakness may be caused by the increased expression of $\gamma$ and $\alpha 7$ nicotinic acetylcholine receptors (nAChRs) on muscle cell membranes, while electroacupuncture could decrease the expression of $\gamma$ and $\alpha 7$ nicotinic acetylcholine receptors. Compared with the rats in control, those treated with immobilization for 14 days showed a significant reduction of tibialis anterior muscle weight, muscle atrophy and dysfunction, which was associated with a significant decrease expression of neuregulin-1 and increased expression of $\gamma$ - and $\alpha 7-n A C h R$ in tibialis anterior muscle. Electroacupuncture significantly enhanced the expression of neuregulin-1 and alleviated the muscle loss, while diminished the expression of $\gamma-$ and $\alpha 7-n A C h R$. Taken together, the beneficial effect of electroacupuncture may be attributed to suppressing $\gamma$ - and $\alpha 7-n A C h R$ production, enhancing neuromuscular function and neuregulin-1 protein synthesis. These results suggest that electroacupuncture is a potential therapy for preventing muscle atrophy during immobilization.

\section{INTRODUCTION}

Muscle atrophy is one of the most important and frequent problems observed in patients in Intensive Care Units [1]. The immobilization therefore is a factor that helps the development of muscular atrophy [2]. In immobilization-related disorders or pathological states, there is loss of muscle mass and decreased muscle strength $[3,4]$. Immobilization changes the function as well as structure of the peripheral neuromuscular system, and alters both protein degradation and synthesis [5]. These have significant clinical consequences, up to and including excess morbidity and mortality. Therefore, effective therapeutic strategies to treat muscle wasting induced by immobilization are urgently required.
Acupuncture is a branch of traditional Chinese medicine that is widely applied to treat various diseases around the world $[6,7]$. Electroacupuncture (EA) is an acupuncture technique that replicates the benefits of exercise through stimulation of muscle contraction. EA has been shown to decrease skeletal muscle atrophy induced by hind-limb suspension in mice [8]. Previous studies have found that EA is a non-pharmacologic approach that can prevent muscle loss induced by chronic kidney disease [9]. The mechanism of EA has been extensively investigated, including increases oxygenation of skeletal muscles and down-regulates the expression of ubiquitinated proteins [10-12]. The mechanism of EA on muscle atrophy, however, has not yet been sufficiently elucidated.

The neuronal $\alpha 7$-type nicotinic acetylcholine receptor $(\alpha 7-n A C h R)$ and the fetal-type $(\gamma-n A C h R)$ 
mRNA encoding could be increased after immobilization has been confirmed [13-15]. In the early fetal stage, the $\alpha 7-n A C h R$ and $\gamma$-nAChR, were scattered throughout the muscle membrane before innervation, while only $\varepsilon$-nAChR was synthesized in muscle cells and was anchored to the end-plate in adults [16]. Our previous study has been shown that the re-expression of $\gamma$ - and $\alpha 7$ $\mathrm{nAChR}$ in mature neuromuscular junction (NMJ) leads to dysfunction and pharmacodynamics changes in nondepolarizing muscle relaxant(s) (NDMRs) in the septic state [17]. However, it remains unknown whether the neuromuscular dysfunction and muscle atrophy induced by immobilization is associated with the re-expression of the $\gamma$ - and $\alpha 7-\mathrm{nAChR}$ in skeletal muscle.

Neuregulin-1 (NRG-1) is a multifunctional protein, it played a significant role in peripheral nerve regeneration and remyelination [18]. Recent studies have established that neuromuscular function was associated with NRG-1 in the sepsis model [19]. Following nerve injury, Schwann cells responds to axonal damage up-regulating NRG-1 and improves remyelination. The NRG-1 plays an important role in this process, thanks to their ability to stimulate proliferation and differentiation of Schwann cells in vitro $[20,21]$.

In this study, we aimed to test the hypothesis that immobilization could decrease the neuromuscular function by increasing $\gamma$ - and $\alpha 7-\mathrm{nAChR}$ expression in the skeletal muscle. We used a typical disuse model that was induced by pining-immobilization to examine the effect of loss of skeletal muscle mass and the changes in the composition of the nAChRs. We also assessed neuromuscular function with hierarchical cluster analysis, principal component analysis and in relation to the expression of $\gamma$ - and $\alpha 7$ subunits of acetylcholine receptors. EA would enhance the NRG-1 signaling pathway, and decrease $\gamma$ - and $\alpha 7-n A C h R$ expression, resulting in suppressed immobilizationinduced muscle loss and alleviated neuromuscular dysfunction. The positive effects of EA could provide

\section{(A)}

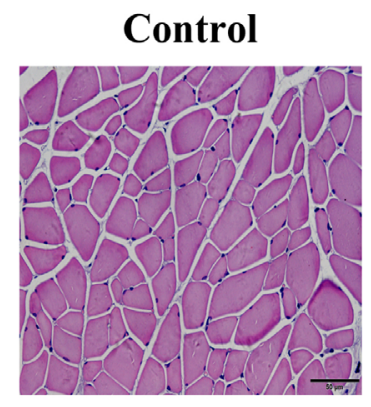

(B)
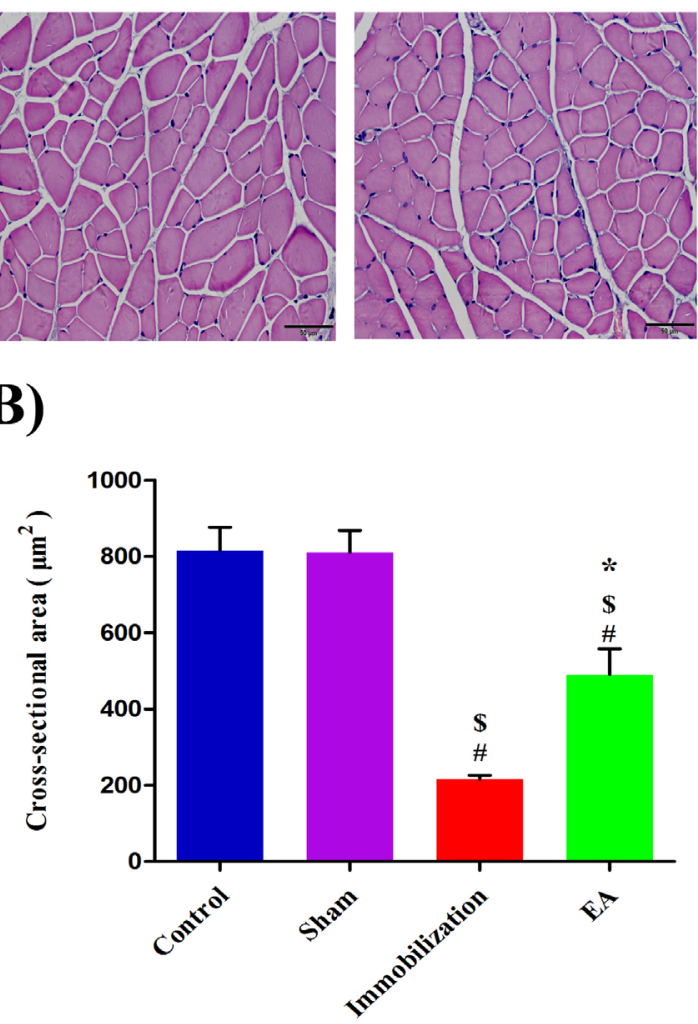

Immobilization

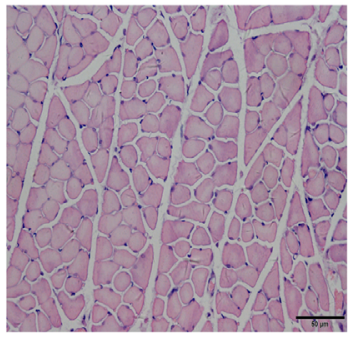

(C)

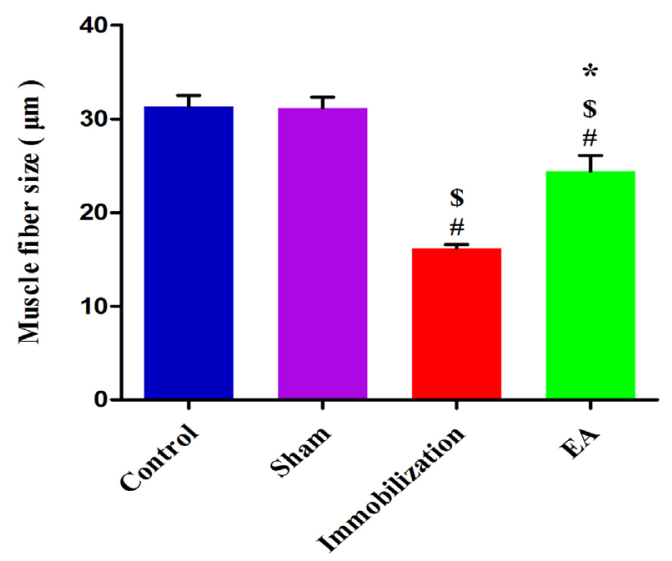

Figure 1: EA prevents immobilization-induced muscle fiber cross-sectional area and muscle fiber size decrease. (A) The morphological changes of tibialis anterior muscle stained with H\&E (magnification, $\times 200$, scale bar $=50 \mu \mathrm{m}$ ) in four groups. (B, C) After 14 days immobilization, muscle fiber Cross-sectional area andmuscle fiber size were significantly decreased in the Immobilization group than in the Control group $(P<0.05)$. With EA treatment, Cross-sectional area andmuscle fiber size were significantly greater in the EA group than in the Immobilization group $(P<0.05)$. The average size of myofibers determined from six mice $\mathrm{x} 10$ sections/mouse/group (Bars: means $\pm \mathrm{SD} ; \mathrm{n}=6$ /group). $P<0.05$ is significant (\# vs. Control, \$ vs. Sham, *vs. Immobilization). 
an additional therapeutic option for treatment of muscle atrophy.

\section{RESULTS}

\section{EA prevents immobilization-induced muscle fiber cross-sectional area and muscle fiber size decrease}

Muscle fiber cross-sectional area was determined in paraffin sections of tibialis anterior muscles using an H\&E staining (Figure 1A). After 14 days immobilization, muscle fibercross-sectional areaand muscle fiber size were significantly reduced in Immobilization group compared with Control group (73\% and $48 \%$ reductions, respectively) (Figure 1B and 1C). Furthermore, immobilization-induced reduction in the cross-sectional area and muscle fiber size was significantly improved in the EA group (127\% and 51\% increase, respectively) (Figure 1B and 1C). EA suppressed the decrease in the muscle fibercross-sectional area and muscle fiber size.

\section{EA prevents immobilization-induced muscle atrophy}

The weights of tibialis anterior muscles in rats with immobilization were significantly less than those of control rats (Immobilization: 322.6 [278.5, 395.3] mg; Control: 729.3 [690.2, 754.3] mg). However, the tibialis anterior muscle weights were significantly higher in rats with EA treatment than in rats with immobilization

(A)

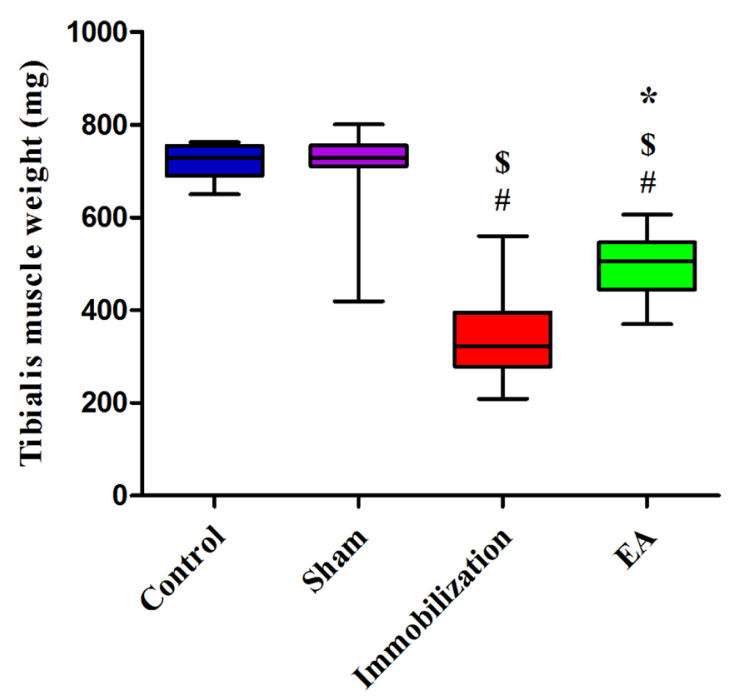

(EA: 505.9 [444.8, 546.2] mg; Immobilization: 322.6 [278.5, 395.3] mg) (Figure 2A). Moreover, the values of muscle wet weight/body weight ratio in the Control and Immobilization group showed the similar trend as the muscle weight. Compared with Immobilization group, EA group have a higher muscle wet weight/body weight ratio (EA: 2.0 [1.8, 2.2], Immobilization: 1.3 [1.1, 1.6]) (Figure 2B). All of this indicated that immobilization induced muscle atrophy and EA treatment prevented immobilization-induced muscle wasting.

\section{EA improves neuromuscular function}

The compound muscle action potential (CMAP) recording of each group was shown in Figure 3A. Amplitude, duration, NCV and twitch tension of CMAP in Control group exhibited no significant difference compared with Sham group. After 14 days of immobilization, amplitude, NCV and twitch tension of CMAP decreased significantly compared with control group (Control: 21.5 [20.2, 23.2] mv, 18.4 [16.5, 20.5] $\mathrm{m} / \mathrm{s}, 17.2[15.5,18.2] \mathrm{g}$, Immobilization: $6.9[4.9,8.8]$ $\mathrm{mv}, 8.8[7.5,10.6] \mathrm{m} / \mathrm{s}, 6.9[6.1,8.5] \mathrm{g})$. However, with EA administration, immobilization-induced reduction in amplitude, NCV and twitch tension were significantly improved in the EA group (Immobilization: 6.9 [4.9, $8.8] \mathrm{mv}, 8.8[7.5,10.6] \mathrm{m} / \mathrm{s}, 6.9[6.1,8.5] \mathrm{g}, \mathrm{EA}: 12$ $[9.2,14.1] \mathrm{mv}, 12.9[10.3,14.8] \mathrm{m} / \mathrm{s}, 11.4[9.4,12.7] \mathrm{g})$ (Figure 3B, 3D and 3E). Furthermore, compared with control group, duration of CMAP in Immobilization group prolonged significantly (Control: 3.8 [3.4, 4.2] ms,

\section{(B)}

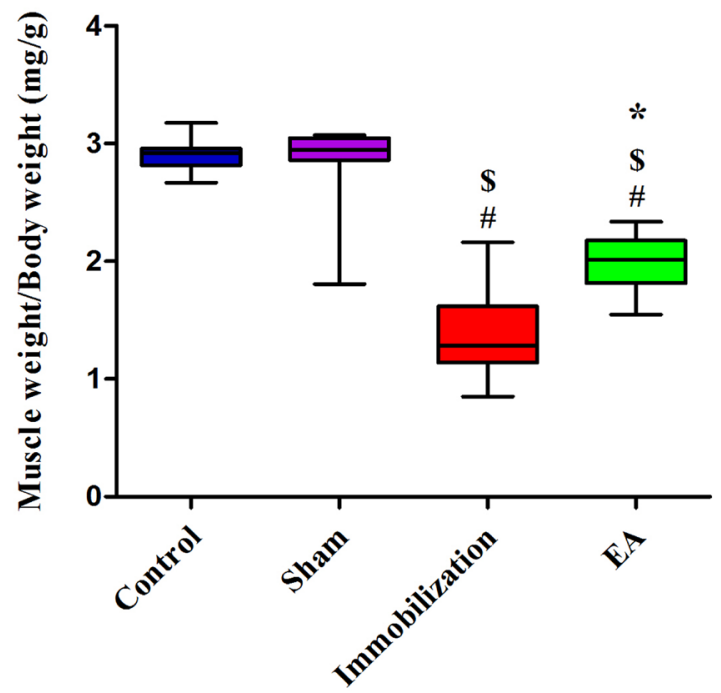

Figure 2: EA prevents immobilization-induced muscle atrophy. (A) Box plot shows Tibialis anterior muscle weight. (B) Box plot shows muscle wet weight/body weight ratio at different groups. (n=8/group). ${ }^{\#} P<0.05$ vs. Control group; ${ }^{\circledR} P<0.05$ vs. Sham group; ${ }^{*} P<0.05$ vs. Immobilization group. 
Immobilization: $5.8[5.3,7.2] \mathrm{ms})$. But EA decreased the duration of CMAP compared with Immobilization group (Immobilization: 5.8 [5.3, 7.2] ms, EA: 5.2 [4.9, 5.7] ms) (Figure $3 \mathrm{C}$ ). All of this results suggested EA significantly improves neuromuscular function.

\section{Hierarchical cluster analysis (HCA)}

The result of the HCA is shown in Figure 4A. This dendrogram suggests four main clusters (Control-C,
Sham-S, Immobilization-Im, EA-EA) with the linkage distance of $0.350,1.618,0.925$ and 13.05 , respectively. The cluster of the Control group and Sham group is very similar with the distance of 3.050, and the next is the Immobilization group and EA group with the distance of 13.05 , respectively. With increasing distance between units, successive branch points represent clusters of increasing size and dissimilarity. The distance between the $($ Control + Sham) group and the (Immobilization + EA) group is 116.8 showing a stronger separation tendency
(A)

Control

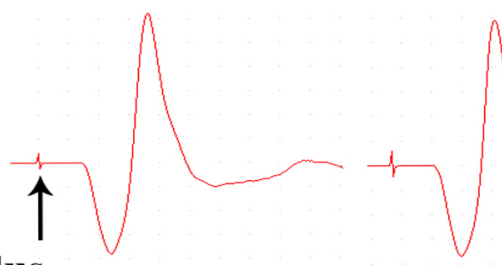

Stimulus

(B)

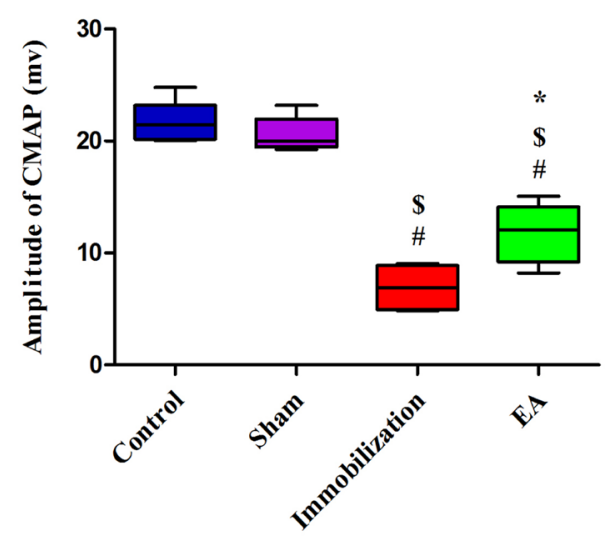

(D)

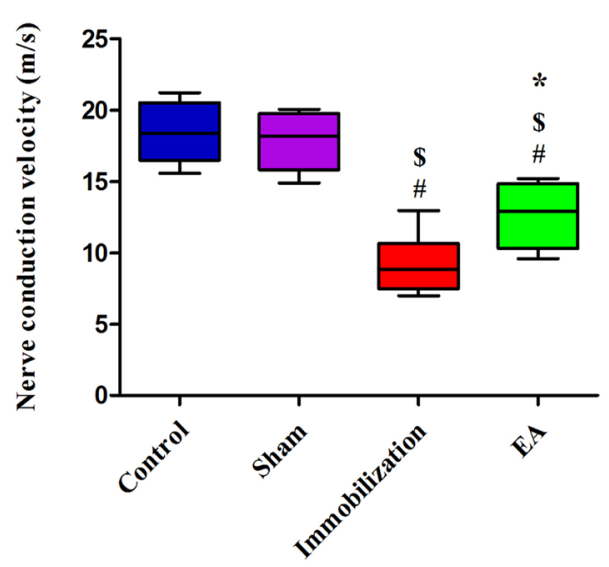

(C)

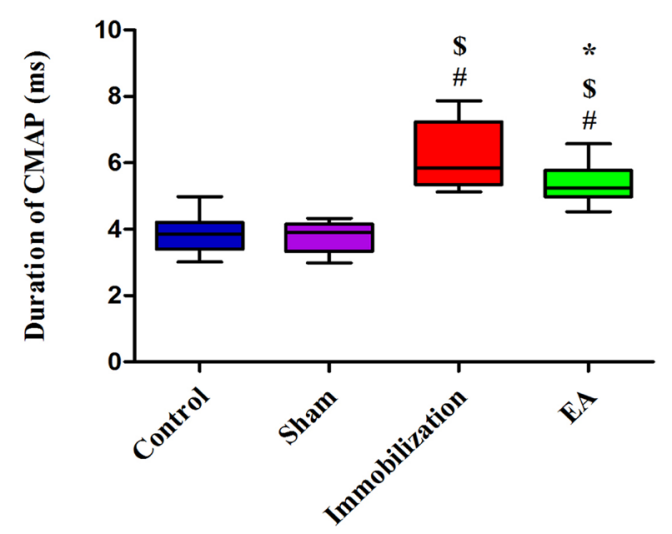

(E)

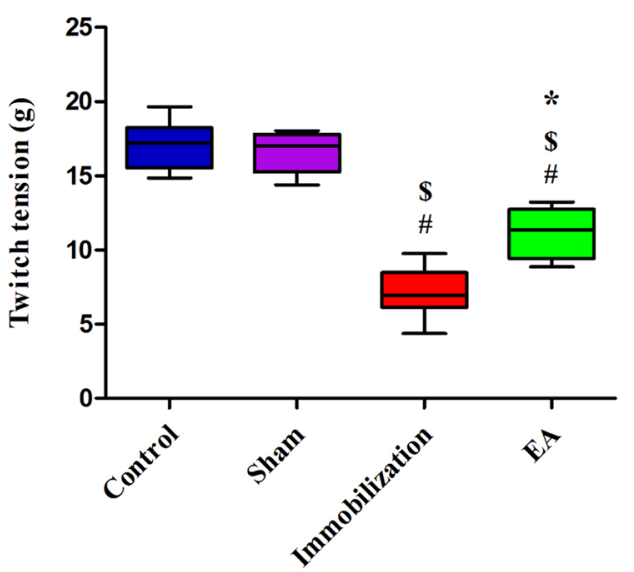

Figure 3: EA improves neuromuscular function. CMAP recorded in the tibialis anterior muscle by needle electrodes after supramaximal needle electrode stimulation of the sciatic nerve. (A) Samples of CMAP recorded in each group, (B) amplitudes, (C) durations, (D) NCV, and (E) single-twitch tension elicited by continuous indirect stimulation. (n=8/group). ${ }^{*} P<0.05$ vs. Control group; ${ }^{\$}$ $P<0.05$ vs. Sham group; ${ }^{*} P<0.05$ vs. Immobilization group. 
of these groups. Therefore, the normal and pathological conditions of neuromuscular function in rats of muscle atrophy could be separated by CMAP data.

\section{Principal component analysis (PCA)}

The neuromuscular function in rats contains fourdimensional parameters of amplitude, duration, NCV and twitch tension, therefore the PCA model is applied to reduce dimension and obtain an overview of the data. Figure 4B shows the PCA scores of neuromuscular function of rats. The $\mathrm{PC} 1$ and $\mathrm{PC} 2$ accounts for $88.3 \%$ and $6.7 \%$ of the total variances, respectively. $\mathrm{PC} 1=0.506 \times$ amplitude $-0.480 \times$ duration $+0.508 \times \mathrm{NCV}+0.51$ $0 \times$ twitch tension. $\mathrm{PC} 2=0.416 \times$ amplitude $+0.797 \times$ duration$0.090 \times \mathrm{NCV}+0.429 \times$ twitch tension. The PCA model shows a clear separation tendency of neuromuscular function of rats in the Immobilization group and the Control group. Meanwhile, neuromuscular function of rats in EA group was distinguished from Immobilization group with the EA treatment indicating EA alleviated immobilizationinduced neuromuscular dysfunction. The obtained results, therefore, support the fact that CMAP data can be used to differentiate normal and pathological conditions of neuromuscular function of rats in muscle atrophy.

\section{EA suppresses the expression of $\alpha 7-n A C h R$ and $\gamma$-nAChR in skeletal muscle of immobilization rats}

The immunofluorescence staining results are shown in Figure 5A and Figure 6A. In control muscle specimens,

\section{(A)}

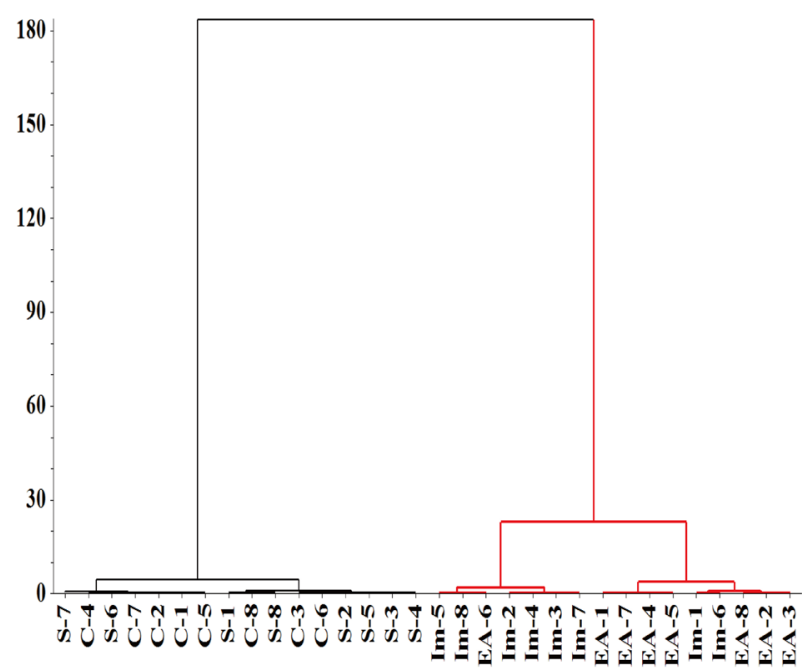

$\alpha 7-\mathrm{nAChR}$ and $\gamma$-nAChR staining were not observed in the skeletal muscle membrane. In the sham group, the skeletal muscle membrane showed up-regulation of $\alpha 7$ $\mathrm{nAChR}$ and $\gamma$-nAChR. A great quantity of $\alpha 7-\mathrm{nAChR}$ and $\gamma$-nAChR accumulated on the entire tibialis anterior muscle membrane after immobilization for 14 days. With EA administration, the expression levels of $\alpha 7-\mathrm{nAChR}$ and $\gamma$-nAChR decreased in the skeletal muscle membrane.

The Western blot analysis results are summarized in Figure 5B and Figure 6B. The protein levels of $\alpha 7$ $\mathrm{nAChR}$ and $\gamma$-nAChR relative to GAPDH significantly increased on immobilization group compared with those in the control and sham group $(P<0.05)$. However, the expression of $\alpha 7-\mathrm{nAChR}$ and $\gamma-\mathrm{nAChR}$ in the tibialis anterior muscle was significantly decreased in the EA group compared with immobilization group $(P<0.05)$, although these levels of improvement were insufficient when compared with the control group.

\section{EA increases the expression of NRG-1 in skeletal muscle of immobilization rats}

Turning to the expression of protein NRG-1 in tibialis anterior muscle, the expression level of NRG-1 was significantly decreased in the immobilization group compared with the control group and sham group $(P<$ 0.05), However, NRG-1 was expressed at higher level in EA group compared with the immobilization group according to Western blot analysis $(P<0.05)$. The results are shown in Figure 7.

\section{(B)}

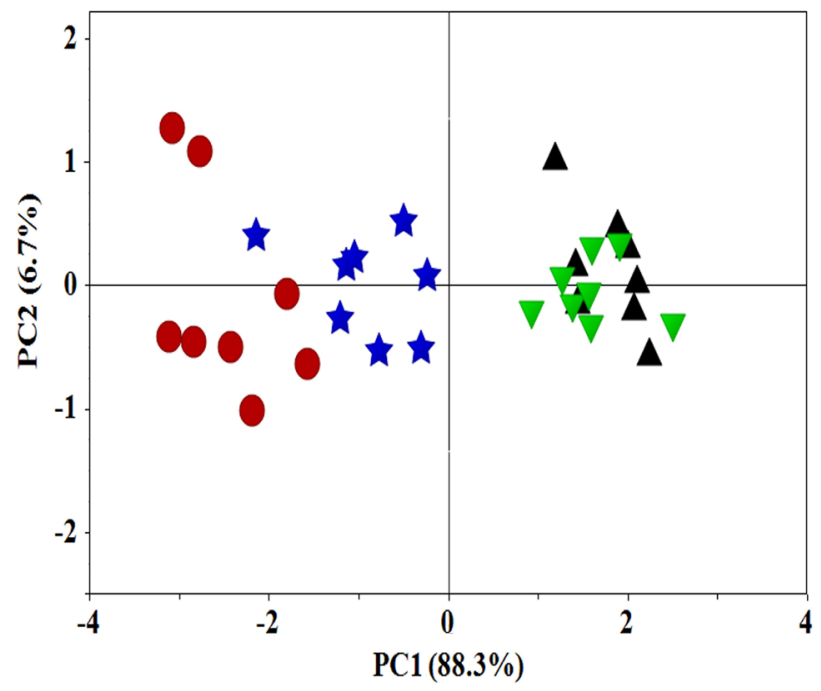

Figure 4: HCA and PCA analysis of neuromuscular function. (A) HCA, Hierarchical cluster analysis of the neuromuscular function of rats. The neuromuscular function of rats in the Control, Sham, Immobilization and the EA group was labeled as C1 C8, S1 S8, $\operatorname{Im} 1 \sim \operatorname{Im} 8$ and EA1 EA8. (B) PCA scores of neuromuscular function of rats. ( $\boldsymbol{\Delta}$, Control group; $\boldsymbol{\nabla}$, Sham group; $\bullet$, Immobilization group; $\star$, EA group; $\mathrm{n}=8$ /group). 


\section{DISCUSSION}

In the current study, we showed that the immobilization induces functional and biochemical changes in skeletal muscle of rats. Immobilization would induce muscle atrophy, and the muscle weakness is associated with increased expression of $\gamma$ - and $\alpha 7-n A C h R$. These changes and characteristics are similar to those in the septic model, including re-expression of immature $\mathrm{nAChR}$, reduction of NRG-1, and decrease of CMAP in tibialis anterior muscles $[11,13]$. The mechanism of the preventive effect of the EA on muscle atrophy may be attributed to suppressing $\gamma$ - and $\alpha 7-n A C h R$ production, enhancing neuromuscular function and NRG-1 protein synthesis.

The rodent model of immobilization has been described previously in rats [22-24], and most recently in mice [25]. Previous methods used to study muscle atrophy have been useful $[26,27]$. However, it would be emphasized that only the immobilization model produced by fixation of joints closely replicates the restricted mobility of critical illness people, reduced neuromuscular
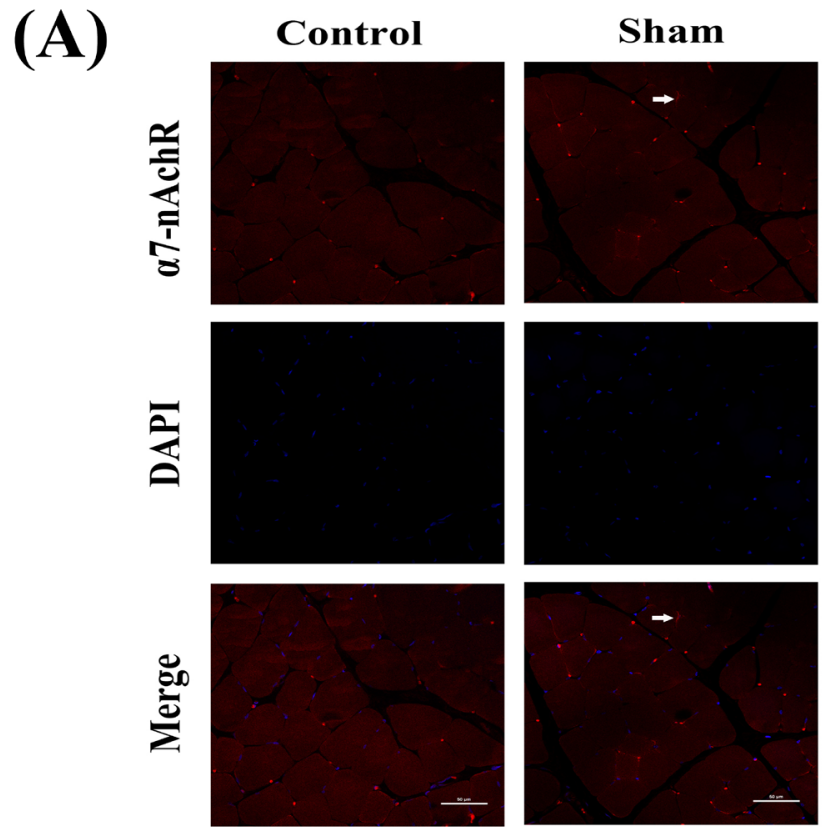

(B)

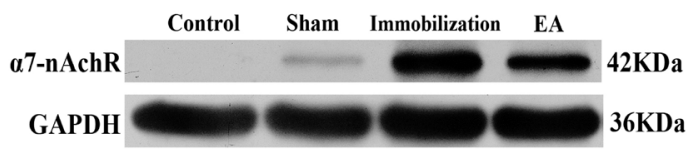

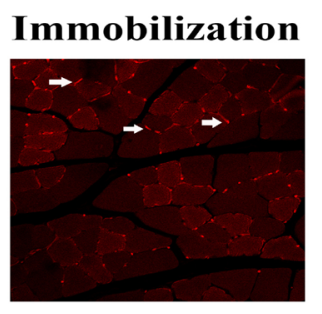
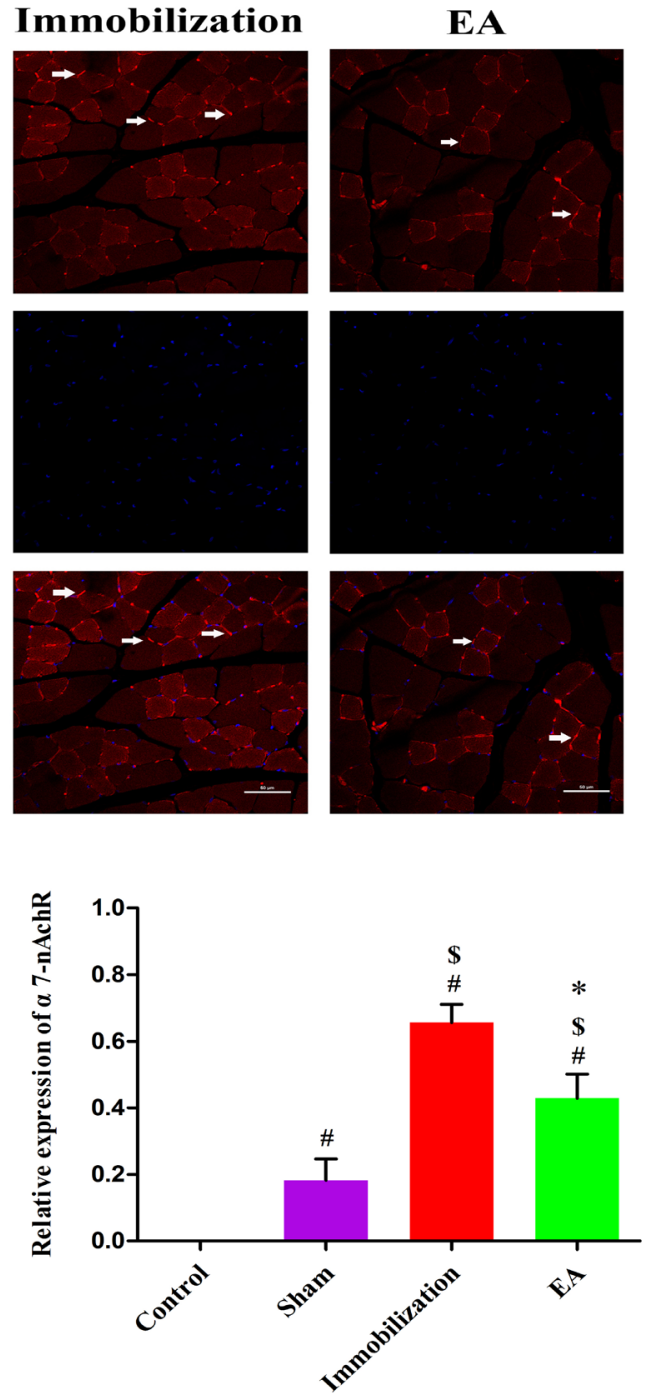

Figure 5: Effects of EA on the expression of $\boldsymbol{\alpha} 7-n A C h R$. (A) Immunofluorescence analysis was performed to determine $\alpha 7$ $\mathrm{nAChR}$ expression on Tiabilis anterior muscle 14 days after the procedure. The samples were immunostained with an anti- $\alpha 7-\mathrm{nAChR}-$ antibody (red, $\rightarrow$ marks a positive expression). The result shows that $\alpha 7-\mathrm{nAChR}$ expression are sharply increased and clustered on the muscle membrane of rats in the immobilization group. Representative results from three independent experiments are shown here (scale bar $=50 \mu \mathrm{m}$ ). (B) The Western blot analysis of the $\alpha 7-\mathrm{nAChR}$ proteins are shown for each groups. Relative intensity of $\alpha 7-\mathrm{nAChR}$ to GAPDH is shown in the graphs. $\alpha 7$-nAChR significantly increased after the immobilization for 14 days. Electroacupuncture suppressed the expression of $\alpha 7$-nAChR in EA group. All values are expressed as means $\pm \mathrm{SD}$ (n=6/group). ${ }^{\#} P<0.05$ vs. Control group; ${ }^{\$} P<0.05$ vs. Sham group; ${ }^{*} P<0.05$ vs. Immobilization group. 
(A)
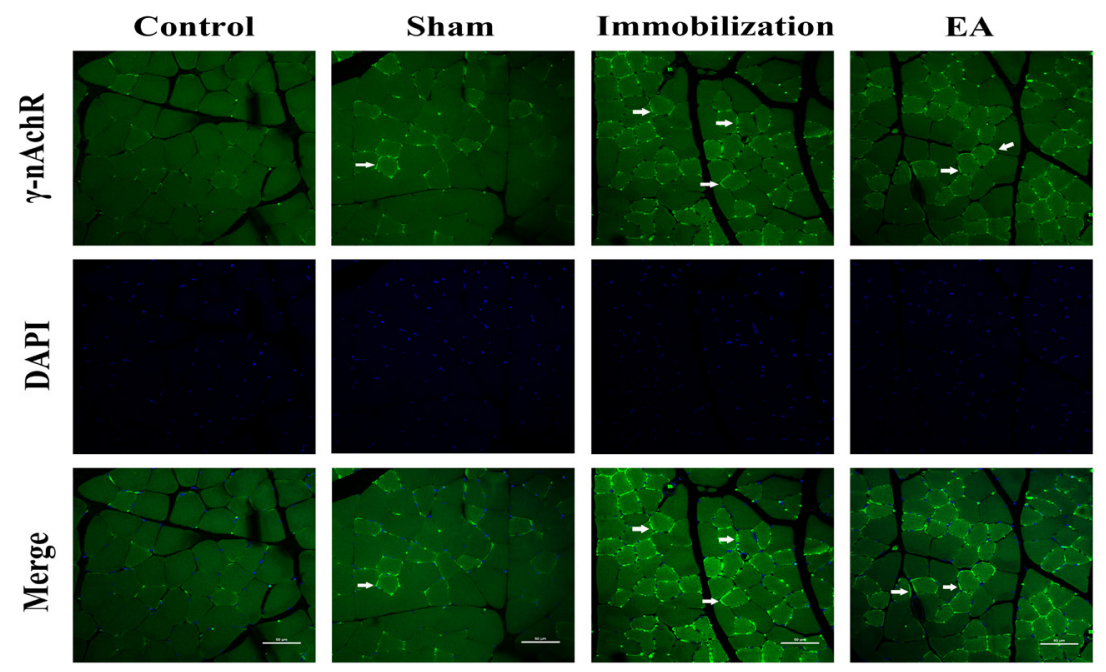

(B)
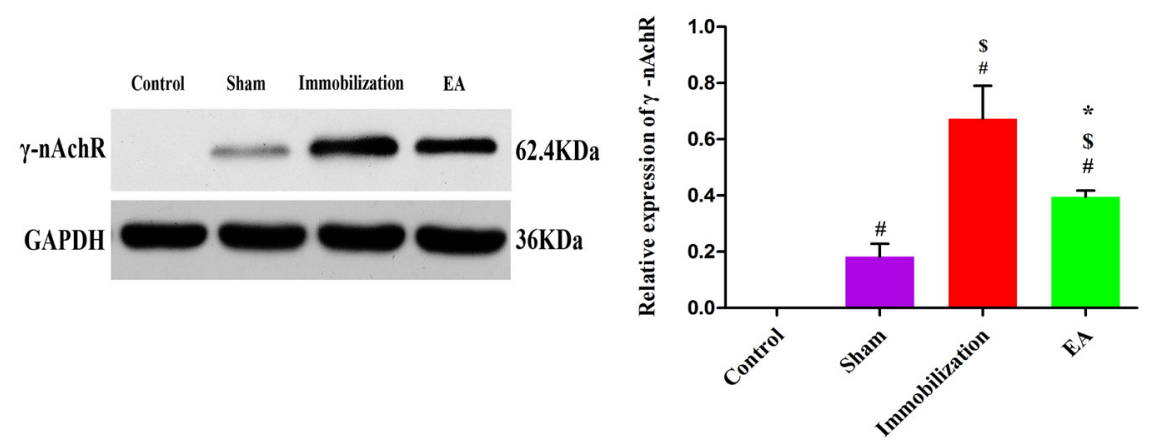

Figure 6: Effects of EA on the expression of $\boldsymbol{\gamma}$-nAChR. (A) Immunofluorescence analysis was performed to determine $\gamma$-nAChR expression on Tiabilis anterior muscle 14 days after the procedure. The samples were immunostained with an anti- $\gamma$-nAChR-antibody (green, $\rightarrow$ marks a positive expression). The result shows that $\gamma$-nAChR expression are sharply increased and clustered on the muscle membrane of rats in the immobilization group. Representative results from three independent experiments are shown here (scale bar $=$ $50 \mu \mathrm{m})$. (B) The Western blot analysis of the $\gamma$-nAChR proteins are shown for each groups. Relative intensity of $\gamma$-nAChR to GAPDH is shown in the graphs. $\gamma$-nAChR significantly increased after the immobilization for 14 days. Electroacupuncture inhibited the expression of $\gamma$-nAChR in EA group. All values are expressed as means $\pm \mathrm{SD}$ (n=6/group). ${ }^{\sharp} P<0.05$ vs. Control group; ${ }^{\$} P<0.05$ vs. Sham group; ${ }^{*} P$ $<0.05$ vs. Immobilization group.
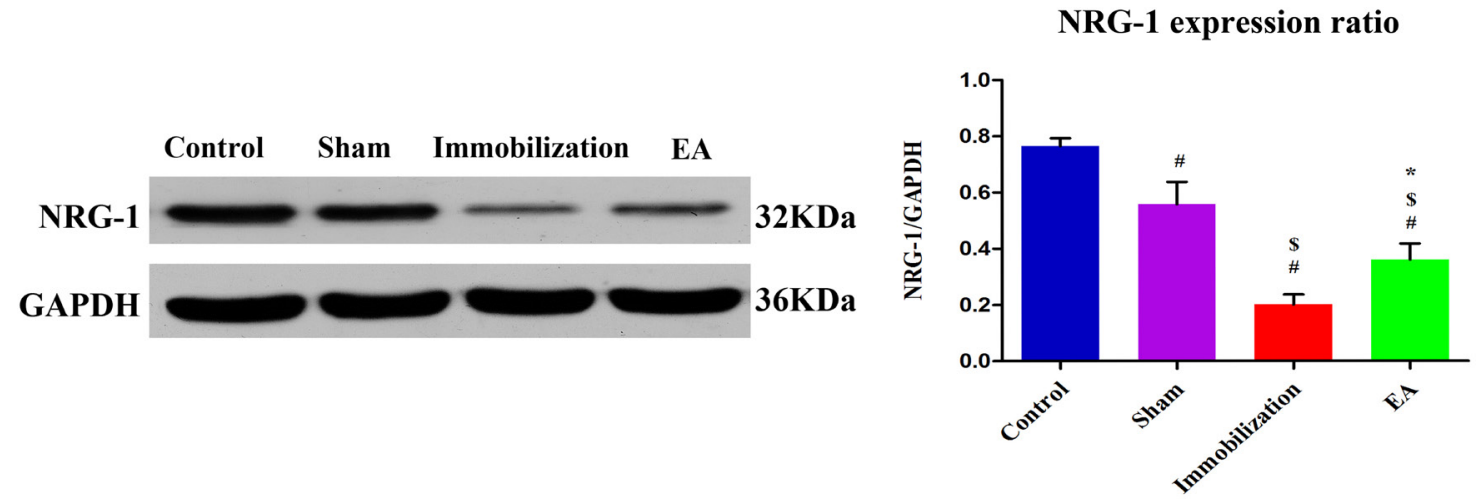

Figure 7: EA activates NRG-1 synthesis in the tiabilis anterior muscle of immobilization rats. Western blot analysis indicated that NRG-1 protein levels in the disuse muscles significantly decreased after 14 days of immobilization compared with the control group $(P<0.05)$. Densitometric analysis showed a significant increase in NRG-1 in the tiabilis anterior muscle after electroacupuncture therapy compared with the immobilization group $(P<0.05)$. The integrated optical density of NRG-1 normalized to integrated optical density of GAPDH. The data are shown as means $\pm \mathrm{SD}$ (n=6/group). ${ }^{\#} P<0.05$ vs. Control group; ${ }^{\$} P<0.05$ vs. Sham group; ${ }^{*} P<0.05$ vs. Immobilization group. 
activity and function changed. Our study has documented the progressive loss of muscle mass with time and the decrease of twitch tension.

The electromyographic examination is a technique for evaluating and recording the electrical activity produced by the skeletal muscle. It is widely used to detect pathophysiology associated with skeletal muscle disorders. Previous studies have shown that disuse weakens aggregate electromyographic activity [28, 29]. In the present study, reduced NCV and prolonged duration time of CMAP were observed 14 days after experimental immobilization. The decline of twitch tension magnitudes elicited by continuous stimulation is concordant with changes of CMAP parameters. Additionally, the HCA and PCA models also demonstrated the normal and pathological conditions of neuromuscular function in rats of muscle atrophy could be separated by CMAP data.

In previous studies, both denervation and immobilization led to the loss of muscle mass and increase in total AChRs on the muscle membrane [30, 31]. As early as the late 1990s, Ibebunjo, C and Nosek, M. T found that immobilization changes the subunits of $\mathrm{nAChR}$, with new $\mathrm{nAChR}(\gamma-\mathrm{nAChR})$ appearing on the muscle membrane [15]. Recently, Khan, M. A and Sahani, N have confirmed that the $\alpha 7-n A C h R s$ were up-regulated 3-fold along with $\alpha 1 \mathrm{AChRs}$ on the immobilized side as compared with the contralateral side after 21 days of immobilization [14], but they failed to identify a link to the altered nAChRs expression. Interestingly, our findings reveal that the $\gamma$ and $\alpha 7-n A C h R$ expression induced by immobilization was similar to that caused by denervation and sepsis. When there is deprivation of neural influence or activity, such as sepsis, burn, or denervation, the immature $\gamma$-nAChR and the neuronal $\alpha 7-\mathrm{nAChR}$, are re-expressed in skeletal muscle. Each of them has different electrophysiological characteristics and affinity for agonists, and it might play a special role in maintaining neurotransmission [32-34].

EA as a therapeutic intervention is widely used in the United States and around the world ${ }^{6}$. Previous studies have demonstrated that acupuncture increases the blood flow in skeletal muscle $[35,36]$ and evokes somatosensory responses of the brain, spinal cord and muscle [37], resulting in a reduction of muscle fatigue. They all have obvious improvement in muscle atrophy, but the mechanism of muscle atrophy is still unclear [38, 39]. In the current study, we determined that EA ameliorates muscle wasting in hind-limb immobilization rats and attenuates $\gamma$ - and $\alpha 7-n A C h R$ re-expression, increasing the NRG-1 expression.

NRG-1 is a key factor in increased transcription of acetylcholine receptors (AChRs) and MuSK and regulates AChRs clusterization [40]. Axonal NRG-1 regulates myelin sheath thickness, which considered a biochemical measure of myelin sheath thickness [41]. Western blot analysis shows the expression of NRG-1 significantly decreased compared with that in the control group.
This result demonstrated that immobilization induces demyelination of nerves, which is concordant with the Electromyography(EMG)findings.

In this study, expressions of NRG-1 decreased after immobilization, which could reduce the inhibitory signals, thereby activating $\gamma$ - and $\alpha 7-\mathrm{nAChR}$ expression. What is more, increases in the expression of NRG-1 were observed in the EA group, These results may illustrate that EA was effective in improving the proliferation of AChRs through regulating NRG-1, leading to muscle fiber regeneration.

Several limitations exit in this study. Firstly, the upstream and downstream pathways associated with EA and NRG-1 expression are not investigated in this experiment. It is possibly associated with the NRG/ ErbB signaling pathway. Secondly, this study revealed the close relation between the EA and $\gamma-, \alpha 7-n A C h R$ changes in immobilization, but the direct causal role of $\gamma$ - and $\alpha 7-n A C h R$ in immobilization need to be validated with genetic manipulations. Further studies are, however, needed to more comprehensively explore this hypothesis.

In summary, the present findings highlight that immobilization decrease the neuromuscular function and activate fetal nAChR $(\gamma-\mathrm{nAChR})$ and neuronal $\alpha 7$ $\mathrm{nAChR}(\alpha 7-\mathrm{nAChR})$ expression. The activation of $\gamma$ - and $\alpha 7-n A C h R$ is associated with the reduction of NRG1. EA suppressed the expression of $\gamma$ - and $\alpha 7-n A C h R$, increased the NRG-1 expression level and improved the neuromuscular function in skeletal muscles. These findings suggest EA is an effective non-pharmacological intervention to alleviate neuromuscular dysfunction and ameliorate skeletal muscle atrophy.

\section{MATERIALS AND METHODS}

\section{Animals and groups}

Male adult Sprague-Dawley (SD) rats (weight range: 240-260g) were acquired from the Experimental Animal Center of Chongqing Medical University (Chongqing, China) and housed in a controlled environment (25 \pm $2^{\circ} \mathrm{C}, 60 \%$ humidity and $12: 12$-h light-dark cycle) for one week with free access to food and water. Based on the Animal Care and Use Committee of Chongqing Medical University, all rats received humane care. The rats were randomly divided into four groups: Control, Sham (Sham group was sham-immobilized by insertion of the hypodermic needles into the bone, which were removed immediately thereafter), Immobilization (Immobilized group was immobilized in one hind limb for a period of 14 days by pinning of knee and ankle joints at 90 degrees using hypodermic needles), and EA (Electroacupuncture treatment was performed for 20 minutes every day for 14 days along with immobilization) ( $n=14$ /group, there were 8 rats used for neuromuscular function observations and the other 6 were used for molecular biological studies). The experiment was approved by the Institution Animal 
Care and Use Committee (IACUC) and carried out according to the Animal Experimentation Regulations of Chongqing Medical University.

\section{Surgical procedures}

The pining-immobilization model, previously described and used in many studies, was used for the current studies [22, 28]. After 1 week of acclimatization, immobilization procedure was performed. The rats were anesthetized with pentobarbital $(60-70 \mathrm{mg} / \mathrm{kg}$ intraperitoneal (i.p.) injection). The knee and ankle joints were immobilized, respectively, by inserting 25 -gauge hypodermic needles through the proximal tibia into the distal femur to produce $90^{\circ}$ flexion at the knee, and 27-gauge hypodermic needles through the calcaneus into the distal tibia to fix the ankle joint at $90^{\circ}$. The sham immobilized limb was subjected to the same manipulations, including boring a hole through the joints but excluding pin insertion to immobilize the joints.

\section{Electroacupuncture (EA) treatment}

EA treatment is administered for 14 days along with immobilization. The rats were kept in specially designed restraint so that they would remain in a recumbent position during EA treatment. EA was performed for 20 minutes every day for 14 days. Acupuncture points were selected according to the WHO Standard Acupuncture guidelines [42]. The positive point (Huan Tiao, GB30) located at the posterior upper border of the hip joint of the hind limbs, vertical needling to a depth of $6 \mathrm{~mm}$. The negative point $(\mathrm{Zu} \mathrm{San} \mathrm{Li}, \mathrm{ST} 36)$ is $5 \mathrm{~mm}$ beneath the capitulum fibulae and $2 \mathrm{~mm}$ lateral to the knee-joint about $7 \mathrm{~mm}$ deep. The needles were connected to an SDZ-II Electronic acupuncture instrument with a $20 \mathrm{~Hz}$ electric frequency using continuous wave, electric current $1 \mathrm{~mA}$. Disposable sterile needles with a diameter of $0.25 \mathrm{~mm}$ (Hua Tuo Medical \& Health Material Co.Ltd. Wuhan, China) were used.

\section{Evaluation of neuromuscular function}

Electromyography (EMG) recordings were measured before and after the immobilization. The data were obtained from the right sciatic nerve that was stimulated supramaximally (intensity $3 \mathrm{~V}$, duration $0.2 \mathrm{~ms}$, and frequency $1 \mathrm{~Hz}$ ) using a direct stimulation electrode (BL-420F; Systems, Inc, Chengdu, China) on the sciatic nerve. Compound muscle action potential (CMAP) was recorded using a superficial disc electrode located on the tibialis anterior muscle before and after immobilization, as described previously [43]. The data were analyzed using the BL-420F USB2.0S (I) version 1.0.2 software Chengdu TaiMeng Instrument Company, Chengdu, China), with amplitude, latency, and duration of CMAP as the primary parameters. The Nerve conduction velocity
(NCV) was calculated as the distance of conduction and/ or latency time. Neuromuscular dysfunction was defined as a decrease by $\geq 20 \%$ of the baseline in the CMAP amplitude [44, 45]. Twitch tension was evaluated using evoked mechano- myography with a nerve stimulator (BL420F; Systems, Inc, Chengdu, China) along with a force transducer. The twitch tension values of the respective tibialis muscle were recorded via an amplifier and displayed using the BL-420F USB2.0S (I) version 1.0.2 software. Anesthesia was maintained with supplemental intermittent doses of pentobarbital $10-20 \mathrm{mg} / \mathrm{kg}$ i.p. injection, empirically administered every $10-20 \mathrm{~min}$. The temperature of rats was maintained at $35.5^{\circ}-37^{\circ} \mathrm{C}$ with a heat lamp.

\section{Tissue preparation}

Before the collection of tissues, all rats were sacrificed by deep anaesthesia with sodium pentobarbital $(65 \mathrm{mg} / \mathrm{kg}$ i.p. injection). Body and muscle wet weights were measured shortly after death, the tibialis anterior muscle specimens of rats were collected in $4 \%$ paraformaldehyde or frozen with liquid nitrogen.

\section{Muscle fiber cross-sectional area and muscle fiber size}

All samples were cut into $8 \mu \mathrm{m}$-thick sections using the LEICA (Wetzlar, Germany, RM2135), alcohol dewaxing and hydration were performed for hematoxylin-eosin (HE) staining. Images were visualized with an Olympus BX51 inverted microscope with a Photonic Science CCD camera (Olympus DP50, Tokyo, Japan). Image-Pro Plus Image (IPP, Version 6.0, Media Cybernetics, USA) software was used to measure the cross sectional area of the muscle fibers and muscle fiber size.

\section{Western blot analysis for $\alpha 7-n A C h R, \gamma-n A C h R$ and neuregulin-1 protein expression}

Tissues were homogenized using lysis buffer (Beyotime, China), and supernatants were collected followed by $5 \mathrm{~min}$ centrifugation of the tissue homogenate at $10,000 \mathrm{rpm}$ at $4{ }^{\circ} \mathrm{C}$. After quantitative analysis of protein concentration, approximately 40 ug of the sample was loaded in each lane for SDS-PAGE electrophoretic separation, transferred to polyvinylidene fluoride membranes (Millipore, Billerica, MA, USA), blocked with $5 \%$ skim milk in Tris-buffered saline for $1 \mathrm{~h}$ at $37.8^{\circ} \mathrm{C}$, and then incubated overnight at $4^{\circ} \mathrm{C}$ with sc-13998 (Santa Cruz, Inc. dilution: 1:500), ab10096 (1:1000), or an antineuregulin (NRG-1) antibody (dilution 1:200, sc-28916, Santa Cruz) as primary antibodies. The membranes were incubated with secondary antibody (1:2000; Beyotime) at room temperature for $2 \mathrm{~h}$. Chemiluminescence Imaging System (Bio-Rad Co. USA) was used to take photos for 
the bands. All results were normalized to glyceraldehyde3-phosphate dehydrogenase (GAPDH) levels.

\section{Immunofluorescence staining for $\alpha 7-n A C h R$ and $\gamma$-nAChR}

An immunofluorescence assay was performed to identify the expression of $\alpha 7$ - and $\gamma$-nAChR in muscles. The endogenous peroxidase was inactivated by incubating the tissue sections in $3 \%$ hydrogen peroxide for $30 \mathrm{~min}$ at $25{ }^{\circ} \mathrm{C}$. The antigen was retrieved using sodium citrate buffer $(0.01 \mathrm{~mol} / \mathrm{L}, \mathrm{pH} 6.0)$ at $96-98^{\circ} \mathrm{C}$ for $10 \mathrm{~min}$; Then, the muscle sections were incubated with $10 \%$ normal goat serum (BosTER Biological Company, Wuhan, China) for $30 \mathrm{~min}$ at room temperature. Then, the $\alpha 7$-nAChR antibody (ab10096, Abcam Ltd. dilution 1:800) and $\gamma$-nAChR antibody (sc-13998, Santa Cruz, Inc., dilution: 1:500) were incubated with the samples overnight at $4{ }^{\circ} \mathrm{C}$. After washing in phosphate-buffered saline, these sections were incubated with fluorescein isothiocyanate-conjugated Goat anti-rabbit IgG antibody to stain for $\alpha 7$-nAChR (dilution 1:200; \#A23320 Abbkine, Inc, California, USA) or Goat anti-rabbit IgG antibody for $\gamma$-nAChR (dilution 1:200; \#A23220 Abbkine, Inc, California, USA) for $1 \mathrm{~h}$ at room temperature to detect each primary antibody. Images were acquired using a Nikon $\mathrm{Al}^{+} \mathrm{R}$ microscope (Nikon, Inc, Tokyo, Japan).

\section{Statistical analyses}

If data were normally distributed the results are displayed as means $\pm \mathrm{SD}$, otherwise as median and the respective $25 \%$ and $75 \%$ quartiles. Significant differences between the groups were analyzed using One-way analysis of variance followed by Tukey's post hoc test if the data was normally distributed. For data with other distributions Kruskal-Wallis followed by a post-hoc test according to Bonferoni method was used. Differences with $P$ values $<0.05$ were considered statistically significant. CMAP data are also analyzed by principal component analysis (PCA) and hierarchical cluster analysis (HCA). The data set is standardized to remove the effect of scaling differences between parameters with unit variance-scaled. PCA and HCA were performed with SIMCA-P software (version13.0, Umetrics, Sweden). All analysis was performed using the statistical software package SPSS 17.0 (IBM Corp, Armonk, NY).

To illustrate the position of the single units in relation to each other, a principal component analysis (PCA) was performed. PCA projects the units from the multidimensional space to a space of lower dimensionality while attempting to preserve the distance relations between them. The first principal component (PC1) accounts for as much of the variability in the data as possible and the PC2 accounts for the next highest levels of variation in declining order. This method also indicates which set of parameters best explains the dissimilarity between groups. To test whether theneuromuscular function of rats can be divided into groups based on CMAP data, HCA was also employed with standardized data set.

\section{ACKNOWLEDGMENTS}

The authors thank Xuechao Hao and Fei Xie for assistance in the study and helpful comments. We also thank Gang Tian from the Department of clinical laboratory of the Chongqing Medical University for his guiding suggestions in data analysis. All authors contributed to the discussion about the results. All authors reviewed the manuscript.

\section{CONFLICTS OF INTEREST}

The authors declare no competing financial interests.

\section{FUNDING}

This work was supported by the National Natural Science Foundation of China (Grant No. 81471282), WU JIEPING Medical Foundation (Grant No. 320.6750.16071) and by the Foundation for Postgraduate Innovative Study of Chongqing (CYS15122). Furthermore, this study was also supported by a grant from National Clinical Key Specialty (Finance No. 2011-170) and Municipal Medical Key Discipline of Chongqing (YuWeiKeJiao No. 2007-2).

\section{REFERENCES}

1. Walsh CJ, Batt J, Herridge MS, Mathur S, Bader GD, $\mathrm{Hu}$ PZ, dos Santos CC. Transcriptomic analysis reveals abnormal muscle repair and remodeling in survivors of critical illness with sustained weakness. Scientific reports. 2016; 6: 29334.

2. Truong AD, Fan E, Brower RG, Needham DM. Bench-tobedside review: mobilizing patients in the intensive care unit from pathophysiology to clinical trials. Critical Care. 2009; 13:4-216.

3. Cohen S, Nathan JA, Goldberg AL. Muscle wasting in disease: molecular mechanisms and promising therapies. Nat Rev Drug Discov. 2015; 14: 58-74.

4. McNally EM, Pytel P. Muscle diseases: the muscular dystrophies. Annual review of pathology. 2007; 2: 87-109.

5. Robinson GA, Enoka RM, Stuart DG. Immobilizationinduced changes in motor unit force and fatigability in the cat. Muscle \& nerve. 1991; 14: 563-573.

6. NIH Consensus Conference. Acupuncture. JAMA. 1998; 280: 1518-1524.

7. Ernst E, White A. The BBC survey of complementary medicine use in the UK. Complementary therapies in medicine. 2000; 8: 32-36. 
8. Onda A, Jiao QB, Nagano Y, Akimoto T, Miyamoto T, Minamisawa S, Fukubayashi T. Acupuncture ameliorated skeletal muscle atrophy induced by hind-limb suspension in mice. BiochemBiophys Res Commun. 2011; 410: 434-439.

9. Su Z, Robinson A, Hu L, Klein JD, Hassounah F, Li M, Wang HD, Cai H, Wang XH. Acupuncture plus LowFrequency Electrical Stimulation (Acu-LFES) Attenuates Diabetic Myopathy by Enhancing Muscle Regeneration. PloS one. 2015;10: e0134511.

10. Ohkubo M, Hamaoka T, Niwayama M, Murase N, Osada T, Kime R, Kurosawa Y, Sakamoto A, Katsumura T. Local increase in trapezius muscle oxygenation during and after acupuncture. Dyn Med. 2009; 16: 8: 2.

11. Fujita N, Murakami S, Fujino H. The combined effect of electrical stimulation and high-load isometric contraction on protein degradation pathways in muscle atrophy induced by hindlimb unloading. J Biomed Biotechnol. 2011; 2011:401493.

12. Tanaka M, Hirayama Y, Fujita N, Fujino H. Comparison of premodulated interferential and pulsed current electrical stimulation in prevention of deep muscle atrophy in rats. J Mol Histol. 2013; 44: 203-11.

13. Fischer U, Reinhardt S, Albuquerque EX, Maelicke A. Expression of functional alpha7 nicotinic acetylcholine receptor during mammalian muscle development and denervation. Eur J Neurosci. 1999; 11 :2856-64.

14. Khan MA, Sahani N, Neville KA, Nagashima M, Lee S, Sasakawa T, Kaneki M, Martyn JA. Nonsurgically induced disuse muscle atrophy and neuromuscular dysfunction upregulates alpha7 acetylcholine receptors. Can J Physiol Pharmacol. 2014; 92 :1-8.

15. Ibebunjo C, Nosek MT, Itani MS, Martyn JA. Mechanisms for the paradoxical resistance to d-tubocurarine during immobilization-induced muscle atrophy. J Pharmacol Exp Ther. 1997; 283: 443-51

16. Albuquerque EX, Pereira EF, Alkondon M, Rogers SW. Mammalian nicotinic acetylcholine receptors: from structure to function. Physiol Rev. 2009; 89 :73-120.

17. Liu L, Min S, Li W, Wei K, Luo J, Wu G, Ao L, Cao J, Wang B, Wang Z. Pharmacodynamic changes with vecuronium in sepsis are associated with expression of $\alpha 7$ and $\gamma$-nicotinic acetylcholine receptor in an experimental rat model of neuromyopathy. Br J Anaesth. 2014; 112: 159-68.

18. Gambarotta G, Fregnan F, Gnavi S, Perroteau I. Neuregulin 1 role in Schwann cell regulation and potential applications to promote peripheral nerve regeneration. Int Rev Neurobiol. 2013; 108: 223-56.

19. Liu L, Xie F, Wei K, Hao XC, Li P, Cao J, Min S. Sepsis induced denervation-like changes at the neuromuscular junction. J Surg Res. 2016; 200: 523-32.

20. Ronchi G, Gambarotta G, Di Scipio F, Salamone P, Sprio AE, Cavallo F, Perroteau I, Berta GN, Geuna S. ErbB2 receptor over-expression improves post-traumatic peripheral nerve regeneration in adult mice. PLoS One. 2013; 8: e56282.
21. Stassart RM, Fledrich R, Velanac V, Brinkmann BG, Schwab MH, Meijer D, Sereda MW, Nave KA. A role for Schwann cell-derived neuregulin-1 in remyelination. Nat Neurosci. 2013; 16: 48-54.

22. Fink H, Helming M, Unterbuchner C, Lenz A, Neff F, Martyn JA, Blobner M. Systemic inflammatory response syndrome increases immobility-induced neuromuscular weakness. Crit Care Med. 2008; 36: 910-6.

23. Mokhtarian A, Lefaucheur JP, Even PC, Sebille A. Hindlimb immobilization applied to 21-day-old mdx mice prevents the occurrence of muscle degeneration. J Appl Physiol. 1999; 86: 924-31.

24. Caron AZ, Drouin G, Desrosiers J, Trensz F, Grenier G. A novel hindlimb immobilization procedure for studying skeletal muscle atrophy and recovery in mouse. J Appl Physiol. 2009; 106: 2049-59.

25. Zhu S, Nagashima M, Khan MA, Yasuhara S, Kaneki M, Martyn JA. Lack of caspase-3 attenuates immobilizationinduced muscle atrophy and loss of tension generation along with mitigation of apoptosis and inflammation. Muscle Nerve. 2013; 47: 711-21.

26. Kandarian SC, Peters DG, Favero TG, Ward CW, Williams JH. Adaptation of the skeletal muscle calcium-release mechanism to weight-bearing condition. Am J Physiol. 1996; 270: C1588-94.

27. Frimel TN, Kapadia F, Gaidosh GS, Li Y, Walter GA, Vandenborne K. A model of muscle atrophy using cast immobilization in mice. Muscle Nerve. 2005; 32: 672-4

28. Fischbach GD, Robbins N. Changes in contractile properties of disused soleus muscles. J Physiol. 1969; 201: 305-20.

29. Hník P, Vejsada R, Goldspink DF, Kasicki S, Krekule I. Quantitative evaluation of electromyogram activity in rat extensor and flexor muscles immobilized at different lengths. Exp Neurol. 1985; 88: 515-28.

30. Yanez P, Martyn JA. Prolonged d-tubocurarine infusion and/or immobilization cause upregulation of acetylcholine receptors and hyperkalemia to succinylcholine in rats. Anesthesiology. 1996; 84: 384-91.

31. Tsuneki H, Salas R, Dani JA. Mouse muscle denervation increases expression of an alpha7 nicotinic receptor with unusual pharmacology. J Physiol. 2003; 547:169-79.

32. Yu CR, Role LW. Functional contribution of the alpha7 subunit to multiple subtypes of nicotinic receptors in embryonic chick sympathetic neurones. J Physiol. 1998; 509: 651-65.

33. Lindstrom JM. Nicotinic acetylcholine receptors of muscles and nerves: comparison of their structures, functional roles, and vulnerability to pathology. Ann N Y Acad Sci. 2003; 998: 41-52.

34. Jonsson M, Dabrowski M, Gurley DA, Larsson O, Johnson EC, Fredholm BB, Eriksson LI. Activation and inhibition of human muscular and neuronal nicotinic acetylcholine receptors by succinylcholine. Anesthesiology. 2006; 104: 724-33. 
35. Noguchi E, Ohsawa H, Kobayashi S, Shimura M, Uchida $\mathrm{S}$, Sato Y. The effect of electroacupuncture stimulation on the muscle blood flow of the hindlimb in anesthetized rats. J Auton Nerv Syst. 1999; 75: 78-86.

36. Ohkubo M, Hamaoka T, Niwayama M, Murase N, Osada T, Kime R, Kurosawa Y, Sakamoto A, Katsumura T. Local increase in trapezius muscle oxygenation during and after acupuncture. Dyn Med. 2009; 8: 2.

37. Ikezono E, Ohama K, Nagayama K, Sawa T, Yoshida T. The effects of acupuncture needling on the evoked responses of brain, spinal cord and muscle in man. Am J Chin Med (Gard City N Y). 1976; 4: 53-9.

38. Agata N, Sasai N, Inoue-Miyazu M, Kawakami K, Hayakawa K, Kobayashi K, Sokabe M. Repetitive stretch suppresses denervation-induced atrophy of soleus muscle in rats. Muscle Nerve. 2009; 39: 456-62.

39. Huang J, Zhang Y, Lu L, Hu X, Luo Z. Electrical stimulation accelerates nerve regeneration and functional recovery in delayed peripheral nerve injury in rats. Eur J Neurosci. 2013; 38: 3691-701.
40. d'Houtaud S, Sztermer E, Buffenoir K, Giot JP, Wager M, Bauche S, Lapierre F, Rigoard P. Synapse formation and regeneration. [Article in French]. Neurochirurgie. 2009; 55 1: S49-62.

41. Michailov GV, Sereda MW, Brinkmann BG, Fischer TM, Haug B, Birchmeier C, Role L, Lai C, Schwab MH, Nave KA. Axonal neuregulin-1 regulates myelin sheath thickness. Science. 2004; 304: 700-3.

42. Lim S. WHO Standard Acupuncture Point Locations. Evid Based Complement Alternat Med. 2010; 7: 167-8.

43. Osuchowski MF, Teener J, Remick D. Noninvasive model of sciatic nerve conduction in healthy and septic mice: reliability and normative data. Muscle Nerve. 2009; 40: 610-6.

44. Preston DC, Shapiro BE. Needle electromyography. Fundamentals, normal and abnormal patterns. Neurol Clin. 2002; 20: 361-96.

45. Van den Bergh PY, Piéret F. Electrodiagnostic criteria for acute and chronic inflammatory demyelinating polyradiculoneuropathy. Muscle Nerve. 2004; 29: 565-74. 